\title{
Configuration of WLAN and Ad Hoc Network Access Point and Research on Internet Topology Control
}

\author{
Aiyong Liu and Hong Chen \\ School of Mathematics and Information Science \& Technology, Hebei Normal \\ University of Science \& Technology \\ liuaiyong2013@163.com
}

\begin{abstract}
Wireless Mesh network, a typical wireless ad hoc network, can effectively solve the "last one thousand meters" problem of broadband access. It, with features of selfforming, self-healing and high bandwidth, provides its users with better service by integrating the advantages of WLAN and ad hoc network and fully utilizing and combining with WiMAX, WiFi and other wireless technologies. In network planning, the effective configuration of access point is of great importance for network set-up cost control. A good topological structure, in addition, plays a decisive role in network throughput capacity. The research stated in the thesis studied the configuration of wireless Mesh network access point and internet topology control, simulating a pure Mesh network connecting to external networks. By studying the relationships between such parameters as gateway connection and broadcast interval with transfer rate of packets, end to end delay and system overhead, their relationships were able to be confirmed and the correctness of the analysis results was tested by the comparative studies of the simulation results.
\end{abstract}

Keywords: wireless Mesh network, configuration of access point, mobile self-forming network, gateway connection

\section{Introduction}

Wireless Mesh network originates from ad hoc network, which was in the very beginning applied in the military and suitable for military applications that were highly demanding but not able to set up large base stations [1]. Ad hoc network nodes can perform the task of forwarding data and, between nodes, one-to-one or one-to-many peer to peer communications. The data can be relayed for many times to its destination and this is what is called ad hoc network. Compared with conventional wired network, the nodes in ad hoc network have the same functions and, unlike other networks that have a control center, have high mobility themselves and all nodes are free to enter or exit the network. The nodes have a variety of paths to forward data, and if there is a node breaking down, the other nodes are able to utilize the nodes left to forward the data [2-3]. Therefore, ad hoc network is particularly robust. These fine qualities of ad hoc network enable it to be used in some situations where a wired network is not able or suitable to be formed. Thus, it is generally used in the military. When used for civilian purposes, the nodes of wireless networks don't necessarily move in a high speed and the network nodes require stable and reliable signals. Wireless Mesh network has many strengths of ad hoc network, including self-forming, self-healing and high speed [4]. Also, as progresses made in such WLAN technologies as IEEE 802.11a, IEEE 802.11 $\mathrm{b} / \mathrm{g}$ and IEEE $802.11 \mathrm{n}$, people have profoundly studied wireless Mesh network. Currently, a number of well-known manufacturers both in China and overseas like Motorola Cisco Systems and Silicon Star have mature products and wireless Mesh 
network solutions put on the market. China's telecom carriers including China Telecom, China Mobile and China Unicom have tested wireless Mesh network in many cities of the country [5-6].

Though wireless Mesh network has a number of advantages compared with conventional wireless network, there are still many problems to be address in its development, among which, wireless network planning is greatly significant for the carriers. Due to restrictions on the locations of wireless network service zone, communications needs of users and technology, consideration should be given to the rational network planning and deployment to achieve a balance between the deployment cost (the number of routers and wireless access point included) and wireless network throughput capacity, to the most cost-effective wireless network set-up to provide good communications service to the users [7], and to how to set up the Mesh router that might influences the whole network capacity and topological structure. Furthermore, wireless technology is making continuous progress and wireless network is adopted in a number of crucial fields. However, this type of network needs reliable and stable network connection, which means that when the network breaks down, wireless network can, still, perform the task of communications. Stock market and healthcare monitoring system, for instance, require the network to continue to work when the access point breaks down. In these fields, whether the access point breaks down or not, wireless network service is needed even if the Internet speed is compromised. Therefore, the research on deployment of fault-tolerant wireless network access point is of significance for improving the reliability and connectivity of wireless Mesh network.

By far, quite a number of scholars have studied the topology control of wireless network. For wireless Mesh network, Mesh nodes can hop several times to extend the coverage of wireless signals by low transmit power so as to optimize the network throughput capacity. Yet, the fast development of mobile terminal and wireless network technology leads to a large increase in users' needs for broadband, making the traffic transmitted between nodes increased. The increasing traffic load of each Mesh node will reduce the throughput capacity of whole wireless network. In addition, topology can cause decisive effects on the performance of wireless ad hoc network, namely, irrational topology can reduce wireless Mesh network capacity, increase the end-to-end delay time of data packets and affect the robustness of node fault tolerance [8]. Sparse topology increases the end-to-end delay, while crowded topology networks affect the network capacity by reducing spatial reuse and increasing interference between wireless nodes. Such problems can be well solved with the utilization of topological structure. Topology control mainly aims to enhance the performance of wireless Mesh network though an effective topological structure such as lowering the hop times of nodes to the gateway and average network load, reducing the effects between nodes and increasing the network throughput capacity. Many studies reveal that controlling transmission power can greatly increase the network throughput capacity, that is, choose proper transmission power according to the propagation distance of nodes rather than the maximum power to transmit messages and increase the channel utilization between nodes. At the same time, lower transmit power can reduce the impacts of signals between nodes and effectively increase system throughput capacity [9-10].

The research stated in the thesis studied the configuration of wireless Mesh network access point and internet topology control, simulating a pure Mesh network connecting to external networks. By studying the relationships between such parameters as gateway connection and broadcast interval with transfer rate of packets, end to end delay and system overhead, their relationships were able to be confirmed and the correctness of the analysis results was tested by the comparative studies of the simulation results. 


\section{Routing of Ad Hoc Network}

Compared with fixed networks, the routing of ad hoc network is much more complex because of the mobile nodes are mobile, the wireless signals that can exhaust, signal interference, one-way signal channel and narrow wireless transmission bandwidth. The early Internet routing protocols were not able to meet these needs. Therefore, specific applications are adopted to deal with the routing protocols of ad hoc network.

Many ad hoc network routing protocols have been put forward in recent years in accordance with the special requirements of wireless ad hoc routing standard. MANET team of IETF now is focusing on ad hoc network routing protocol and has come up with a number of drafts such as zone routing protocol (including ZRP and AODV protocols). Besides, scientists have also published articles related to ad hoc network routing protocol and put forward many ad hoc network routing protocols like DSDV. There are mainly three types of routing protocol according to routing-triggering theory, that is, table-driven routing protocol, on-demand driven routing protocol and hybrid routing protocol.

\section{Internet Gateway in Interconnect Structure}

Wireless communications and Internet technology have made people's life more convenient. Now, people have a higher standard for real-time communications and more users need Internet connection when going out. Because of the wireless communications signal channel that might exhaust, the influences between signals and noises, WLAN, from the perspective of network layer, is still one of the single-hop networks, in which the communications between nodes relies on the central node that has a small range of signal coverage and lower mobility. Therefore, it will cost a large amount of money to satisfy people's needs for Internet connection by just adding a large number of nodes [12].

Wireless ad hoc network is an independent network without central nodes and the network system has no fixed infrastructure and routers. All the nodes can move at any point and connect dynamically to other nodes in any form without being affected by the surroundings. Under such circumstances, wireless multiple-hop network can be set up by integrating the characteristics of wireless ad hoc network with mobile IP, making it possible for mobile agents to communicate with foreign agents. As long as one of the ad hoc network nodes is within the coverage area of foreign agents, the other nodes are able to connect with the gateway by the way of multi-hopping and exchange information via gateway with the mainframe of the Internet.

\subsection{The Gateway Interconnecting Ad Hoc Network and the Internet}

All data will be transmitted to the gateway if the mobile nodes of ad hoc network want to exchange information with the mainframe of the Internet. The role of the gateway is to connect ad hoc network to the Internet fixed network. Therefore, the gateway has to realize bilateral network protocol stack. In physical layer and data link layer, ad-hoc nodes implement such specified protocols as IEEE802.11 and HIPERLAN/2, and AODV, DSR and other ad hoc routing protocols in network layer. Network layer can be divided into two sublayers. IP is implemented in upper sublayer and is on the top of the sublayer in which non-IP, ad hoc routing protocol is implemented. IP data packets are transmitted by the upper sublayer and the encapsulated data packets are received by the lower sublayer in which such protocols as TCO and SLP are implemented [13]. At the same time, the bilateral network protocol stack, that is, fixed Internet network and mobile ad hoc network, is implemented on the router serving as the gateway. In mobile ad hoc network, the network nodes are responsible for implementation of ad hoc routing protocol and reception of data packets 
and two different routings are implemented. The higher layer protocols can be implemented via the gateway, enabling data exchange between different protocols. The nodes in ad hoc network, by forwarding and translating the data, can communicate with the mainframe of the Internet.

\subsection{Relevant Interconnect Signaling}

To interconnect different networks, interconnect gateway with ad hoc network and Internet protocol stack is used in constructing the network and the communication between mobile nodes in ad hoc network and Internet nodes is realized by message forwarding, reception and transmission of the gateway [15]. This presents a way of interconnecting ad hoc network and the Internet, showing how the mobile nodes communicate with the gateway. For example, reactive gateway discovery mechanism in ad hoc network adopting AODV routing protocol is implemented when originating nodes find and expand message format, the signaling format of RREQ to be extended transmitted by wireless nodes to the gateway.

Expanded RREQ signaling format is basically the same as the original one except that an I is added in the head of messages, which is called global Internet address approach. Adding $I$ in the head of messages means that wireless nodes in ad hoc network want to communicate with all nodes in the world, connect to the gateway by transmitting the messages and exchange information with some nodes in the Internet at the same time, which is different from the communication with nodes in independent ad hoc network.

Similarly, except the I added in the head of messages, the signaling format replied by the gateway is basically the same as the original one. If there is an I in the head of RREP message, it means that the message comes from the gateway. Therefore, the extended RREP signaling message plays the role of ICMP of Internet protocol in replying messages.

\section{Experiment and simulation}

Figure 1 shows the structure of a simulated network, from which, it can be seen that server_1 is wired server providing various application services like FTP, HTTP, VOIP and video conference, that gateway_1 is the gateway connecting the server to multi-hop network, that station nodes represent the nodes in wireless Mesh network which can receive data from server_1, the wired server. The nodes here can not only perform the task of receiving data, but play the role of jumping or relaying to transmit data to other nodes. The whole network structure shows a wireless Mesh network. 


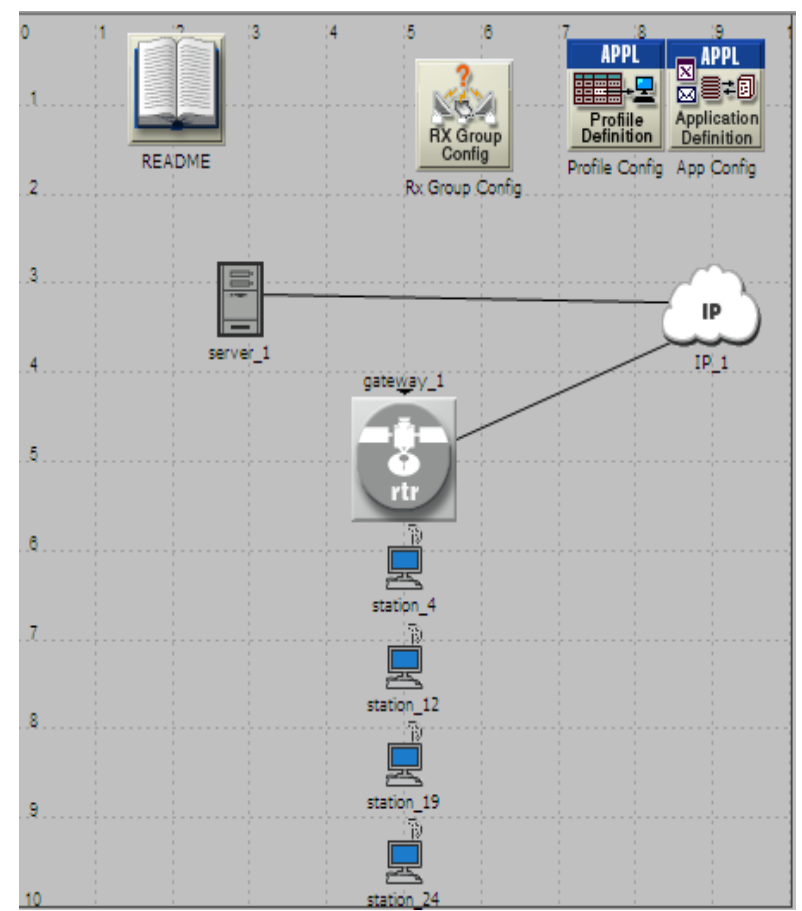

Figure 1. Simulated Network Structure

Figure 2 demonstrates the Ftp traffic sent from server_1. The abscissa stands for time and the simulated time is 10 minutes. The ordinate shows the traffic sent measured in units of bytes/sec. As can be seen from Figure 2, congestion window experienced great fluctuations when in multi-hop network.

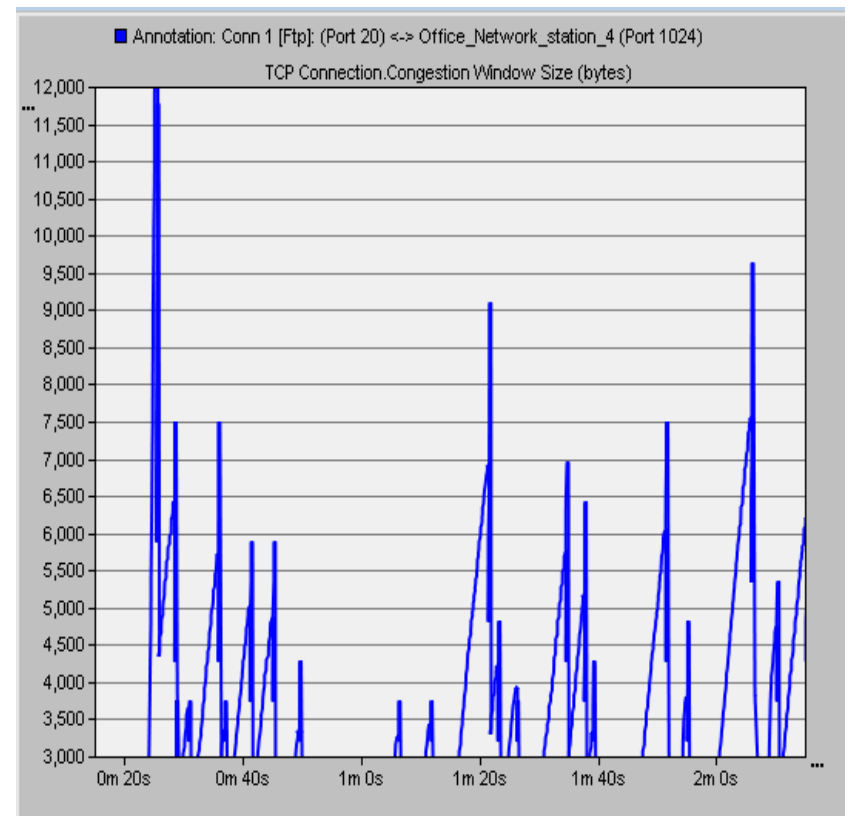

Figure 2. Ftp Traffic Sent from Server_1

Figure 3 shows the time each node spent to transmit files of same size. The abscissa stands for simulated time lasting for 10 minutes. The ordinate represents the time each node used to download data measured by second. It can be seen from Figure 3 that the time the first node spent to forward data from other nodes was the longest and that the 
time the second node costed was the shortest because it forwarded less data than the first one and was closer to the access point than the third and fourth node.

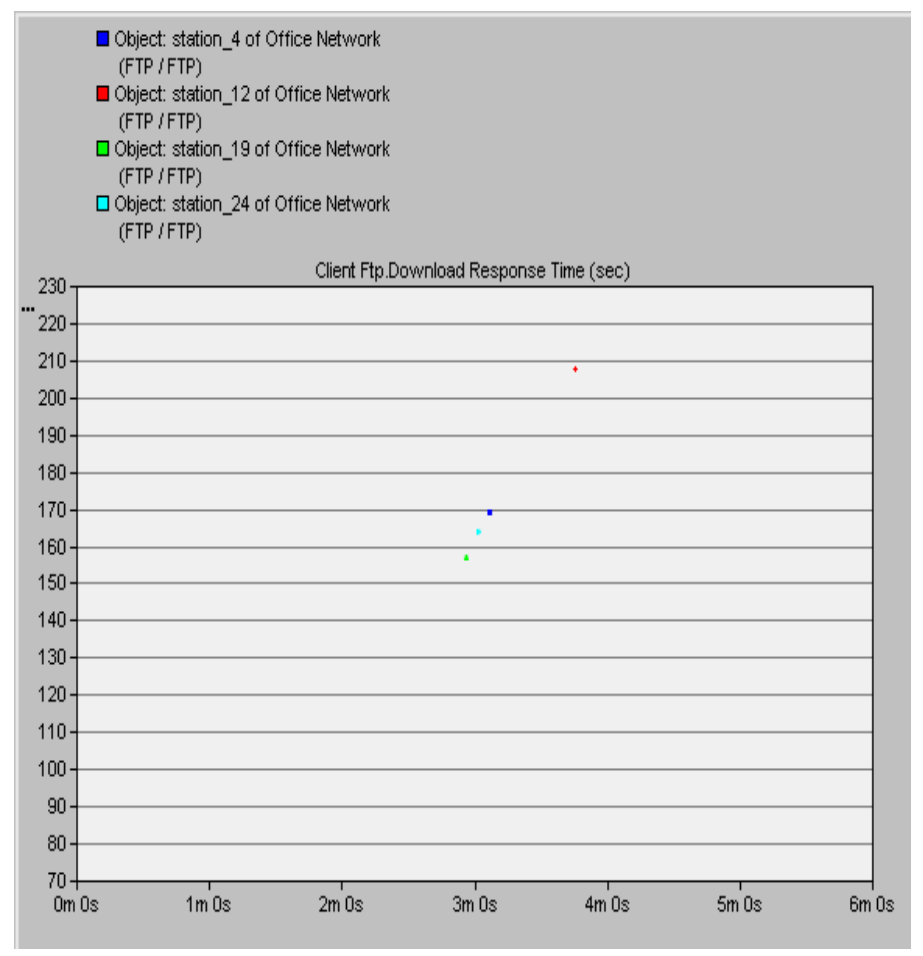

Figure 3. Time Each Node spent to Download Files of Same Size

Figure 4 shows a node downloading a file. The abscissa stands for the download time measured by minute. The ordinate stands for the time spent to download data measured by second.

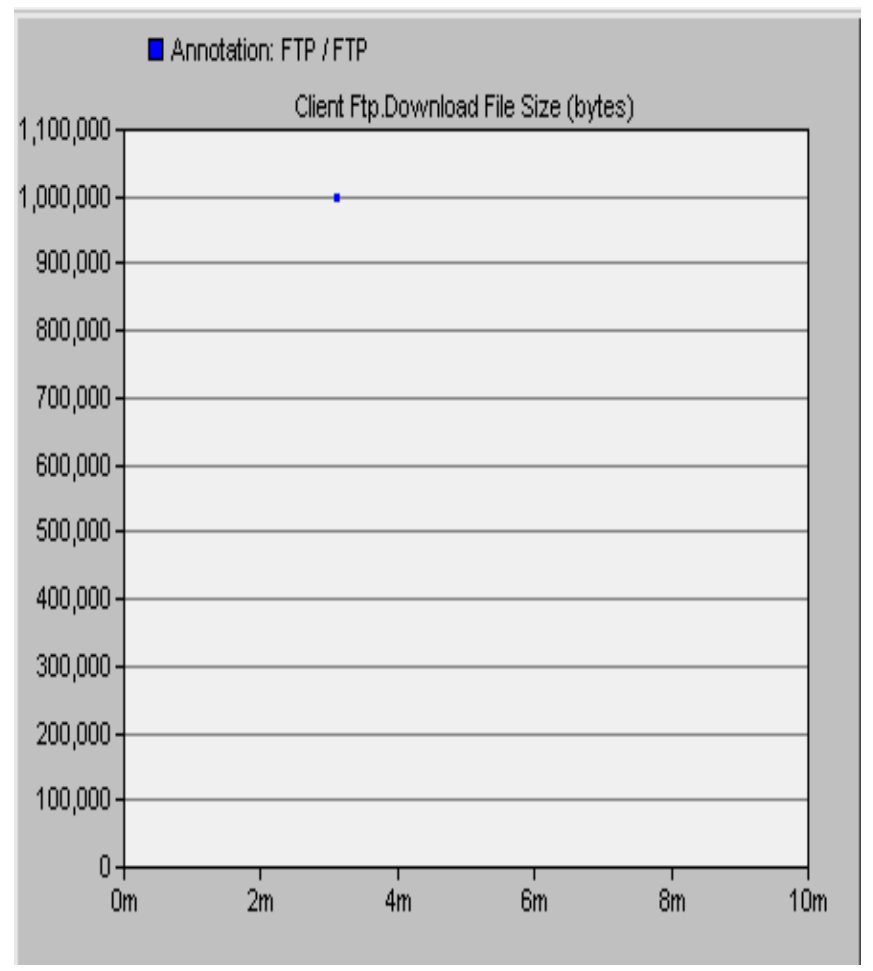

Figure 4. A Node Downloading File 
Figure 5 shows each node transmitting files of same size. The abscissa stands for time used measured by minute and the ordinate the size the file transmitted measured by byte. Each node transmitted the file at the same time, but when it finished, the time node 19 spent was the shortest followed by node 24 , node 4 and node 12 . The underlying reason is the location of the nodes and that some nodes have to forward the data from other nodes.

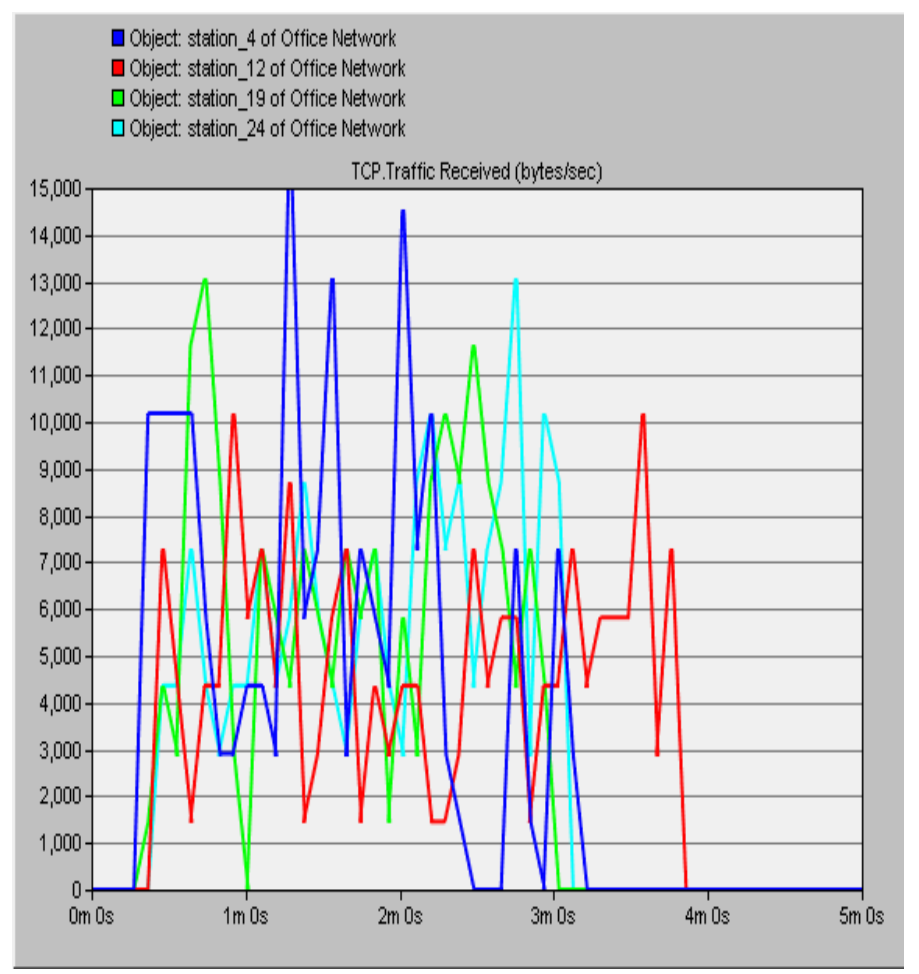

Figure 5. Nodes Downloading Files

As wireless network develops and becomes popular, the solution of accessing the last one thousand broadband has under heated discussion. Wireless network has distinguished itself with its many advantages. The goal of wireless network configuration is to meet users' needs for communication and within the coverage of the network, reduce the configuration cost even if it means a reduction in the number of access point. Actually, wireless access point might break down on many occasions and lead to interrupted service, which is unacceptable for some important applications. Therefore, fault tolerance must be considered in the configuration of a wireless network.

When wireless signals transmit data, they, in the process might be affected by the medium and other obstacles and interference might occur between nodes. Wireless network topology has great impacts on network throughout capacity, therefore, how to improve network performance with effective topology control and power control has been heatedly discussed by scholars. 


\section{References}

[1] Bogdanov, E. Maneva, Riesenfeld S. Power-aware base station positioning for sensor networks [A] In: proceedings of the Twenty-third Annual Joint Conference of the IEEE Computer and Communications Societies (INFOCOM04) [C], 2004: 575-585

[2] M. Kamenetsky, Unbehaun M. Coverage planning for outdoor wireless LAN systems [A]. International Zurich Seminar on Broadband Communications Access, Transmission, Networking [C], 2002

[3] Youngseok Lee, Kyoungae Kim, Choi Y. Optimization of AP placement and channel assignment in wireless LANs [A]. Local Computer Networks, 2002 Proceedings LCN 2002 27th Annual IEEE Conference on [C], 2002: 831-836

[4] Xiang Ling, Yeung K L. Joint access point placement and channel assignment for 802.11wireless LANs [J]. IEEE Transactions on wireless communications, 2006, 5(10): 2705-2711

[5] Gandhi R. Tolerance to access-point failures in dependable wireless local-area networks [A]. In: proceedings of the Ninth IEEE International Workshop on Object-Oriented Real-Time Dependable Systems [C]. 2003: 136-143

[6] K. Wu, W. Liao. Interference Efficient Topology Control in Wireless Ad Hoc Networks [J]. IEEE CCNC 2006, Volume 1, Jan. 2006

[7] P.V. Rickenbach, S. Schmid, R. Wattenhofer. A Robust Interference Model for Wireless IEEE International, April 2005

[8] M. Malekesmaeili, M. Shiva, Soltan M. Topology Optimization for Backbone Wireless Mesh Networks [A]. Fifth Annual Conference Communication Networks and Services Research (CNSR '07) [C], 2007: 221-230

[9] G. Li L L Y, W. S. Conner, Sadeghi B. Opportunities and Challenges for Mesh Networks Using Directional Antennas [A]. WiMesh workshop [C], 2005:

[10] P. Bahl, J. Y. Halpern, L. Li Y M W, et al. Analysis of a Cone-Based Distributed Topology Control Algorithm for Wireless Multi-hop Networks [A]. In: proceedings of the twentieth annual ACM symposium on Principles of distributed computing [C]: ACM, 2001: 264-273

[11] D. Blough M L, G. Resta, P. Santi. The K-Neigh Protocol for Symmetric Topology Control in Ad Hoc Networks [A]. In: proceedings of the 4th ACM international symposium on Mobile Ad Hoc networking and computing (MOBIHOC'03) [C]: ACM, 2003: 141-152

[12] M. Burkhart, P. Von Rickenbach, R. Wattenhofer, et al. Does Topology Control Reduce Interference? [A]. In: proceedings of the 5th ACM international symposium on Mobile Ad Hoc networking and computing (MobiHoc'04) [C], 2004: 9-19

[13] R. Prim. Shortest connection networks and some generalizations [J]. The Bell System Technical Journal, 1957: 1389-1401

[14] X.Y. Li, K. M. Nejad, W. Z. Song. Interference-Aware Topology Control for Wireless Sensor Networks [A]. In: proceedings of the Second Annual IEEE Communications Society Conference on Sensor and Ad Hoc Communications and Networks(SECON) [C], 2005: 263-274

[15] R. Wakikawa, J. T. Malinen, C. Perkins. Global Connectivity for IPv6 Mobile Ad Hoc Networks. IETF Internet Draft, 2003-10. 